\title{
Percutaneous endoscopic lumbar laminectomy
}

\author{
Yukoh Ohara', Sumito Shimizu², Junichi Mizuno' \\ ${ }^{1}$ Center for Minimally Invasive Spinal Surgery, ShinYurigaoka General Hospital, Kawasaki, Kanagawa 215-0026, Japan . \\ ${ }^{2}$ Spine and Spinal Cord Center, Omigawa General Hospital, Katori-shi, Chiba 289-0332, Japan.
}

Correspondence to: Dr. Yukoh Ohara, Center for Minimally Invasive Spinal Surgery, ShinYurigaoka General Hospital, 255 Furusawa Asao-ku, Kawasaki, Kanagawa 215-0026, Japan. E-mail: yukoh@juntendo.ac.jp

How to cite this article: Ohara Y, Shimizu S, Mizuno J. Percutaneous endoscopic lumbar laminectomy. Mini-invasive Surg 2017;1:74-80.

\author{
Article history: \\ Received: 05-03-2017 \\ Accepted: 20-03-2017 \\ Published: 30-06-2017 \\ Key words: \\ Percutaneous endoscopic lumbar \\ laminectomy, \\ lumbar canal stenosis, \\ single port endoscope
}

\begin{abstract}
Aim: Percutaneous endoscopic lumbar laminectomy or laminotomy (PELL) is a minimally invasive surgical technique to treat lumbar canal stenosis. The procedure is undertaken using a single port endoscope, as with percutaneous endoscopic lumbar discectomy (PED). PED has become popular with spinal surgeons in Japan as a suitable surgery for lumbar disc herniation patients. Because PED has the powerful advantage of structural preservation, it allows for short hospital stays and early recovery of the patient. PELL and PED are conceptually very similar, in that they are both minimally invasive. PELL is not as popular as PED, however. The aim of the current study was to explore the reasons why. Methods: The current study reports the early experiences of surgeons at this institution in using the PELL technique, and its limitations. Results: The goal of PELL is total flavectomy and decompression of the bony structure. Currently, there are difficulties and limitations in achieving decompression using PELL with small tools. Conclusion: PELL requires much more skill than PED and the learning curve is not steep. PELL is minimally invasive for the patient, but further developments of the endoscope or procedures are required to achieve widespread use.
\end{abstract}

\section{INTRODUCTION}

Percutaneous endoscopic surgery for spinal degenerative diseases is carried out using a special single-port endoscope under irrigation, making the invasiveness of this surgery extremely low..$^{[1-5]}$ Percutaneous endoscopic lumbar discectomy (PED) has been extensively reported, and the development of drills and bipolar coagulators has broadened its application. ${ }^{[6-10]}$ In particular, the ability to use drills has expanded the surgical indications for the interlaminar approach (PED-IL) to include patients with an insufficiently wide interlaminar space and those with concomitant osseous stenosis. ${ }^{[11,12]}$ Attempts have been made to use PED-IL to perform posterior decompression for lumbar canal stenosis (LCS), in a procedure referred to as percutaneous lumbar laminectomy (or laminotomy) (PELL). However, no studies concerning this method using a single-port endoscope have yet been published. This report describes the PELL procedure and its limitations, based on the initial clinical experiences of surgeons at this institution.

\section{METHODS}

\section{Surgical indication}

Currently, PELL is only performed in this institution to treat LCS at a single vertebral level. In the case of multiple stenosis, after obtaining consent from the

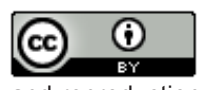

and reproduction

For reprints contact: service@oaepublish.com

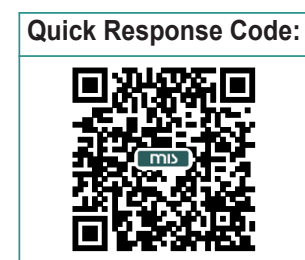


patient, single-level decompression is only performed if it is likely to affect the patient's symptoms; if the patient requests treatment of all the vertebral levels potentially causing the symptoms, microscopic surgery is performed instead. This procedure is used to treat all types of central canal stenosis and lateral canal stenosis.

\section{Surgical instruments}

A special single-port endoscope is used for PELL, as with PED. In this institution, a scope $7 \mathrm{~mm}$ or $8 \mathrm{~mm}$ in diameter is used (VERTEBRIS, Winnova Richard Wolf Medical Instruments Corporation, Germany). The 7-mm endoscope has an 8- $\mathrm{mm}$ sheath and is easily manipulated, even in a narrow interlaminar space, but is incompatible with some of the instruments that can be used for an 8-mm endoscope. An 8-mm endoscope can be used with drill sizes up to $3.5 \mathrm{~mm}$, which is useful for drilling large areas of bone. The 8-mm endoscope also enables the use of a larger Kerison punch, as well as curved and curved basket punches (Winnova Richard Wolf Medical Instruments Corporation, Germany). The 8-mm endoscope is easier to use at first, until proficiency in the procedure has been achieved. A special drill (Primade 2; Nakanishi, Japan), and a bipolar flexible radiofrequency probe (Elman Trigger-Flex probe; Elman International) are also used.

\section{Surgical procedure}

As PELL requires a longer operation time compared with PED, PELL is currently performed under general anesthesia. In most cases, the approach is performed on the side with the most prominent symptoms, but the opposite side may be chosen if preoperative images indicate that decompression of the osseous stenosis is likely to be easier. If the operator is right-handed and no laterality of the symptoms is present, an approach from the left is used as it allows the surgeon to control the endoscope to drill the lower edge of the upper lamina easily. Discography is not performed if only the posterior component should be decompressed, but if disc manipulation may be required, discography is undertaken from the opposite side. After set-up of the equipment, a frontal fluoroscopic image is used to check the extent of decompression during the procedure. Physiological saline is used for irrigation, which is delivered at low pressure through instillation from a height of $30-40 \mathrm{~cm}$ above the operating table.

A 7-mm skin incision is made just beside the spinous process under the affected level. An obturator is advanced from this location along the base of the spinous process above the lamina below, as far as the interlaminar space. In patients with a narrow interlaminar space and a thick ligamentum flavum, the obturator cannot be inserted into the interlaminar space. Therefore, obturator and sheath should be inserted until they reach the bone surface. However, if the obturator is not inserted deeply along the surface of the bone, the soft tissues will be more difficult to deal with after endoscope insertion. In this institution, a bevel-type sheath at a $30^{\circ}$ angle is typically used.

After the soft tissues have been dealt with, drilling is initiated at the center of the superior edge of the lamina under the lesion [Figure 1]. Epidural fat tissue may persist at this site even in patients with severe LCS, meaning that the depth of the epidural space can be safely confirmed. After confirming the epidural space, drilling is performed as far as the attachment of the ligamentum flavum on the approach side of the lamina under the lesion, to enable the dissection of the ligament at its attachment.

The superior facet process is then also drilled, and the stenosis of the lateral recess on the same side is treated in this step. If the ligamentum flavum cannot be detached from its attachment, its complete removal is difficult. As only limited kinds of instruments can be used, flavectomy cannot be performed unless either the attachment of the ligamentum flavum at the lamina is dissected using a drill, or laminotomy itself is carried out as far as the attachment of the ligament, as in a conventional lumbar surgery. Debulking of the ligamentum flavum can carried out using a punch or basket punch. Therefore, the bone shape and the lesion responsible for the symptoms should be established using preoperative images.

The use of a single-port endoscope makes it difficult to perform a flavectomy on both sides, particularly a superolateral flavectomy on the contralateral side. If the insertion angle of the endoscope is limited, making manipulation on the opposite lateral side problematic, the base of the spinous process of the superior lamina must first be drilled to secure the pathway for insertion. If widespread laminotomy of the ipsilateral lamina above the lesion is required, drilling is easier if a straight-type sheath is initially used, as this helps to prevent soft tissue from entering the sheath. Once a sufficient interlaminar space has been obtained, switching to a $30^{\circ}$ bevel or duck-bill type sheath for subsequent operations is necessary to treat the opposite side, as these cannot be carried out using a straight-type sheath. When changing the sheath, an obturator is inserted as a guide.

\section{Postoperative management}

Hemostasis of bleeding from soft tissue and resected 

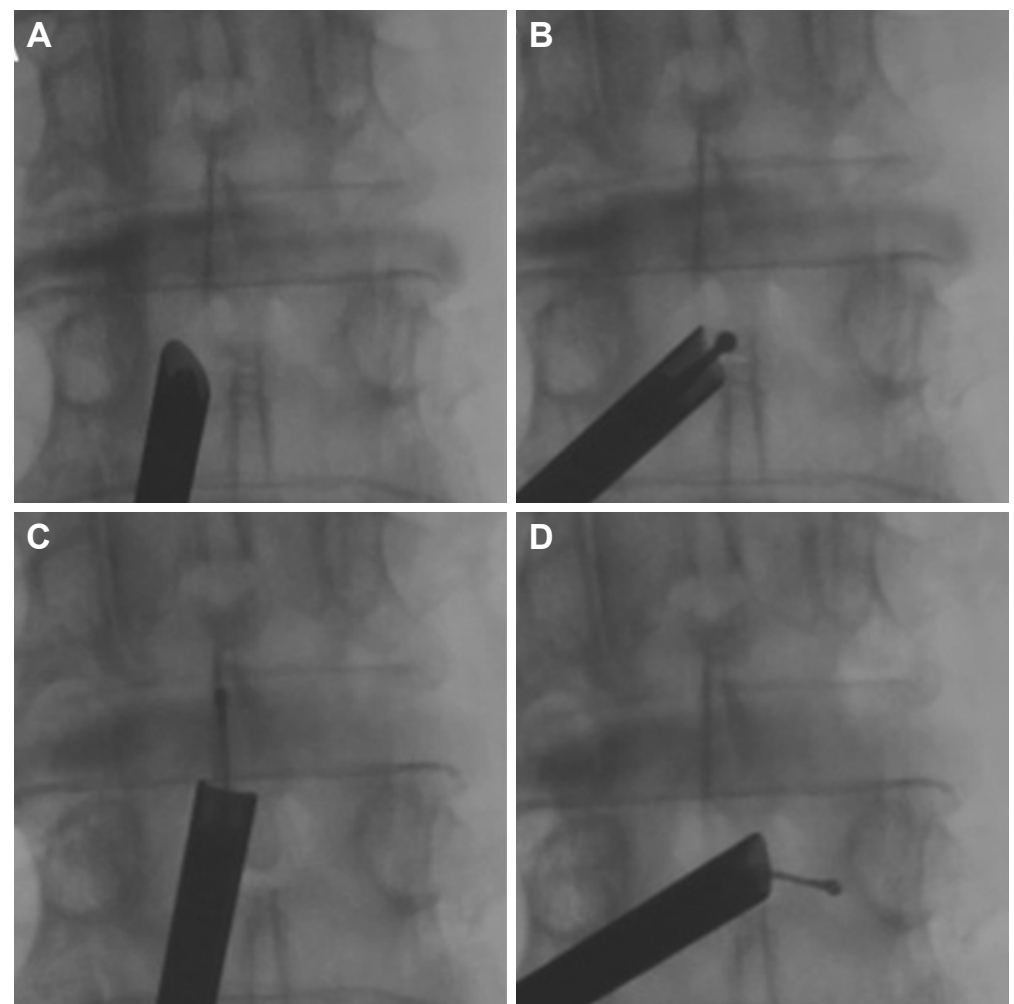

Figure 1: Intraoperative frontal fluoroscope images. A: Insertion of 30-degree bevel-type sheath on the obturator; B: starting point of laminectomy with drill; C, D: confirmation of decompressed area

bone stumps can be achieved using a bipolar coagulator. However, the decompressed area after this surgery is very narrow. As in other minimally invasive surgeries, there is no large space to avoid dural compression if a small hemorrhage occurs. A negative-pressure drain is therefore used. The amount of postoperative fluid drainage is only approximately $10 \mathrm{~mL}$, but dull pain in the legs may persist for around a week after drain removal in some cases, possibly as a result of leachate or tiny hematomas. After the endoscope has been withdrawn, the drain tube is advanced inside the sheath and placement of the tip is confirmed using fluoroscopy.

\section{RESULTS}

Using PELL for the treatment of LCS has some advantages compared with conventional surgery. First, PELL requires a small skin incision and produces less muscle damage, thereby resulting in a shorter hospital stay. Second, the greatest advantage of this technique is the good field of view on the opposite side, as once the superior tip of the lamina has been drilled, the opposite side lateral recess can be decompressed. After decompression, the transverse root is visible as far as the vicinity of the intervertebral foramen. Drilling of the lateral recess can be carried out relatively easily. However, training is needed for this method, because of the limited kinds of operative tools.

\section{Complications}

In addition to the same sort of dural damage that may occur during conventional surgery, other potential complications include elevated intracranial pressure caused by a long period of high-pressure irrigation, as may also occur in PED As previously described, irrigation is delivered at comparatively low pressure, and the risk is not great in the absence of complications such as dural damage. The treatment of dural laceration varies depending on its size. If the damage is minor, cerebrospinal fluid leakage is not a problem, because of the narrow surgical space. However, a laceration that exceeds $2 \mathrm{~mm}$ and includes the arachnoid membrane may lead to nerve root herniation, causing pain, and will require treatment. As in conventional surgery, caution is required with respect to dural adhesion. Although the wide variety of instruments used in conventional surgery cannot be employed in dissection, this procedure does enable direct visual observation. Areas that cannot be viewed must be treated with greater caution.

\section{Illustrated cases}

Case 1

A 76-year-old woman had been attending this hospital for several years complaining of pain in the left leg. Pain was also present at rest, over an area in the left $L 5$ region. Intermittent claudication with numbness in both legs 


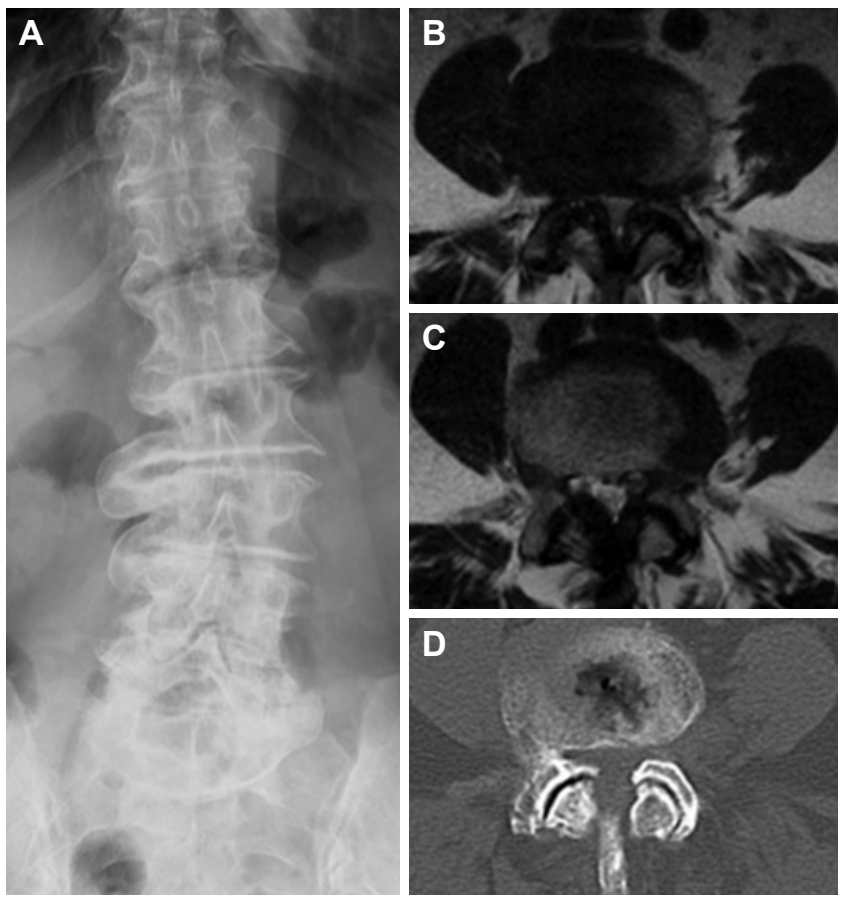

Figure 2: Preoperative neuroimages reveal degenerative lumbar scoliosis and remarkable bilateral canal stenosis with hypertrophied ligamentum flavum and superior facet joint at L4/5. A: Roentgram $(A-P) ; B, C$ : magnetic resonance imaging $T 2 W I$ axial image at $L 4 / 5$; D: computed tomography axial image at L $4 / 5$
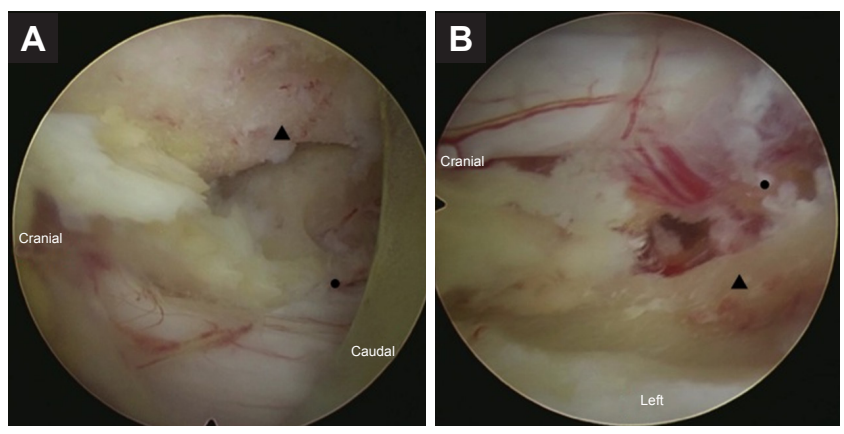

Figure 3: Intraoperative endoscopic images (left side approached percutaneous endoscopic lumbar laminectomy). A: Contralateral view. Right side is the caudal of the patient. Triangle: facet joint (already drilled out) of right side; dot: right side L5 nerve root. B: Ipsilateral view. Left side of the figure is the caudal of the patient and lower side is the left side of the patient. Triangle: residual superior facet which medial side was drilled out to decompress the L5 root; dot: left L5 root

was also evident when she walked for approximately $10 \mathrm{~m}$. Preoperative neuroimaging revealed severe LCS at the $L 4 / 5$ level caused by a hypertrophied ligamentum flavum and superior facet, and degenerative scoliosis was present [Figure 2]. PELL was performed via a left approach, and bilateral decompression was performed [Figure 3]. The operation was finished after confirmation of the decompressed area by using frontal fluoroscope. The negative pressure drain tube was inserted and confirmed the position by frontal fluoroscope. Postoperatively, the pain improved. Dull
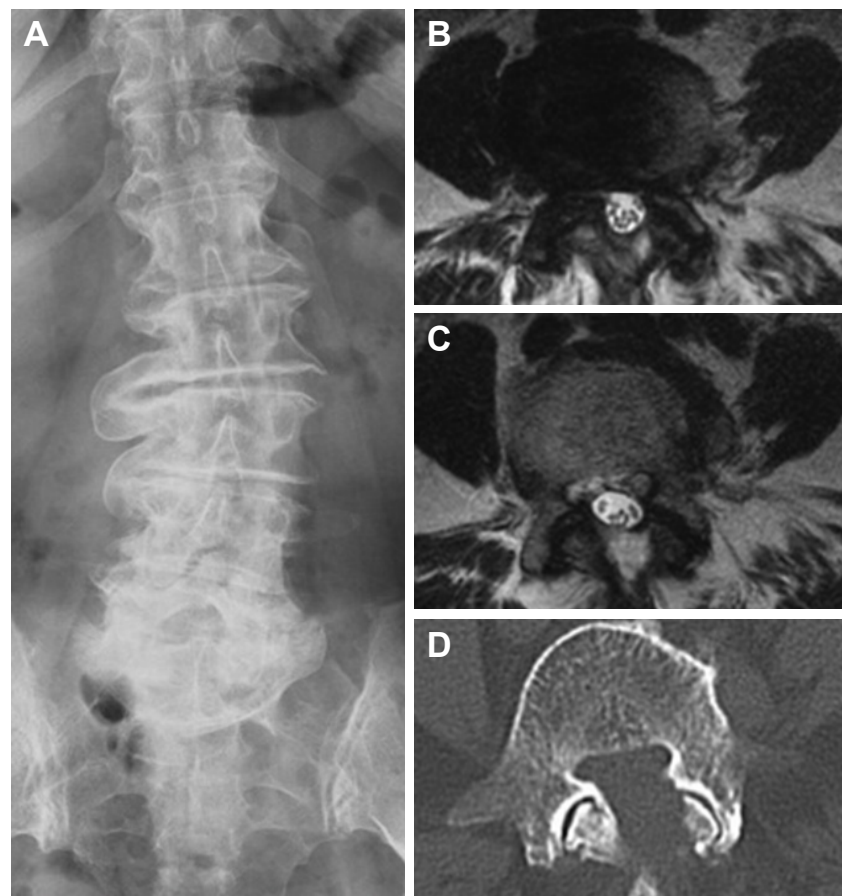

Figure 4: Postoperative neuroimages show reasonable decompression of the spinal canal at the L4/5 level and no deterioration of the scoliotic change. A: Roentgram $(A-P)$; $B$, $\mathrm{C}$ : magnetic resonance imaging $\mathrm{T} 2 \mathrm{WI}$ axial image at $\mathrm{L} 4 / 5$; $\mathrm{D}$ : computed tomography axial image at $L 4 / 5$

pain was present for several days after drain removal, but this pain improved to 0 on a visual analog scale at postoperative day 7 . Postoperative neuroimaging showed that adequate decompression had been achieved [Figure 4]. Six months after surgery she could walk without any limitation and all sensory disturbance was gone.

\section{Case 2}

A 48-year-old man developed pain in the right leg after lifting a heavy object at work. Treatment using conservative therapy for 3 months had not produced any improvement. The painful area was in the L5 region, and was aggravated by load-bearing on the right side. Preoperative neuroimaging revealed stenosis of the right lateral recess caused by ossification of the posterior longitudinal ligament (OPLL) and an ossified ligamentum flavum (OLF) at the L4/5 level [Figure 5A and B]. Preoperatively discography was performed with left side needle insertion. PELL with a right-side approach was performed, and the OLF was drilled out and the hypertrophied ligamentum flavum was resected. After the decompression of the posterior elements, the subligamentous disc was resected and the OPLL under the L5 root was drilled out. The lesion under the theca can not be drilled out but the compression of the right L5 root was improved. The operation was finished with the negative pressure 

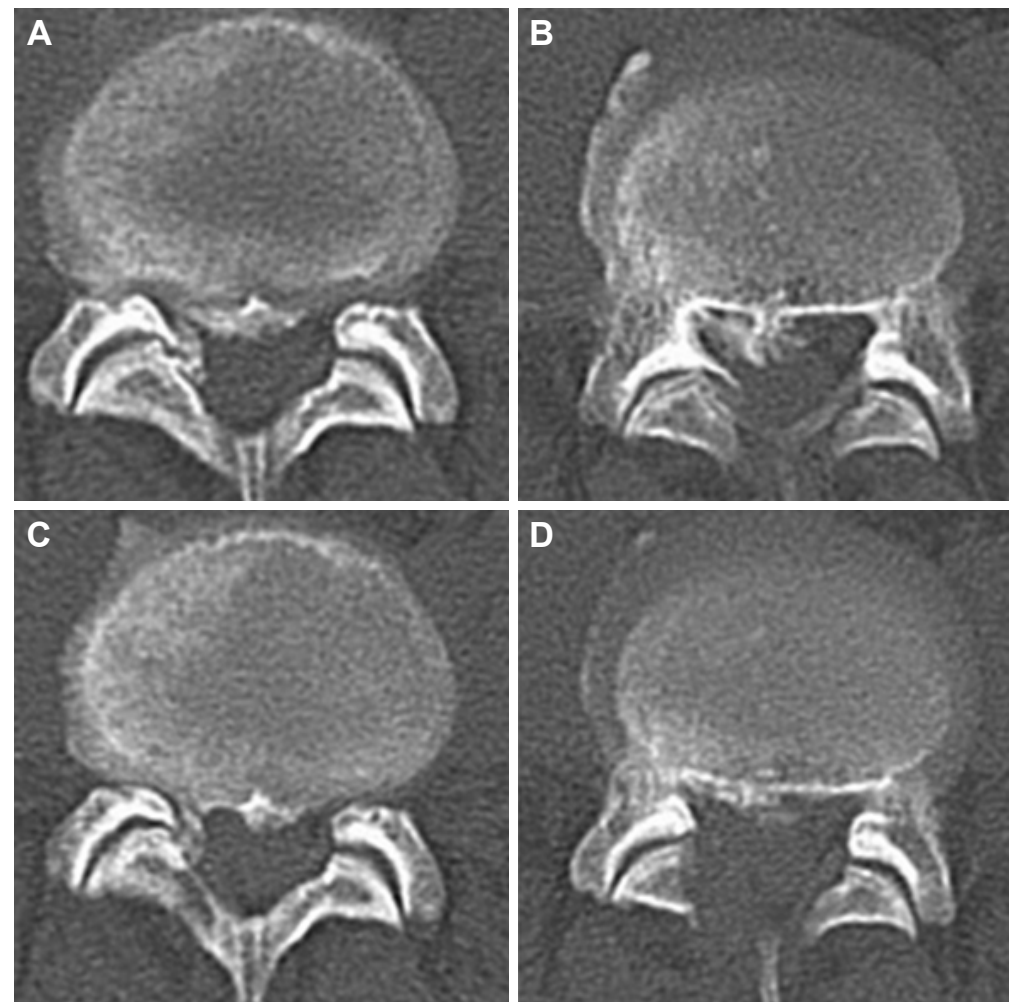

Figure 5: Preoperative computed tomography (CT) images (A, B) at L4/5 show right side ossification of ligamentum flavum and ossification of posterior longitudinal ligament. Postoperative CT images (C, D) at the same level show reasonable decompression of the spinal canal

drain insertion. Postoperatively, the pain completely improved, and numbness also resolved after 2 weeks. Postoperative imaging revealed good decompression [Figure $5 \mathrm{C}$ and D].

\section{DISCUSSION}

The gold standard for surgical treatment of LCS is bilateral laminotomy, medial facetectomy, and flavectomy using a microscope. ${ }^{[13]}$ Although some studies have stated that bilateral decompression via a unilateral approach is less invasive, ${ }^{[14]}$ this approach may cause muscle damage through detachment of the muscles attached to the spinous processes. Microendoscopic laminectomy (MEL) is another method that causes less muscle damage and enables a visual field to be obtained on the opposite side by changing the orientation of the tube retractor. ${ }^{[15,16]}$ However, this procedure is associated with problems such as a higher incidence of dura mater damage and other complications. ${ }^{[17,18]}$ Furthermore, intraoperative fogging of the camera with blood and other fluid requires cleaning of the camera on a regular basis. ${ }^{[16]}$

In PED IL, if LCS is located on the approach side, then lateral recess decompression is performed together with discectomy. In cases with central lumbar canal stenosis, PELL is used as an additional option to perform decompression on the opposite side. As with PED, this method minimizes the destruction of tissue during the surgical approach and entails less tissue invasion than the MEL approach described previously. However, in practice it has not yet become as popular as PED. The main reason for this is the difficulty of the surgical procedure. ${ }^{[19,20]}$ PELL requires the use of limited kinds of small instruments to perform decompression entirely within the interlaminar space. Although operations on the same side enable exposure of the attachment of the ligamentum flavum by expanding the extent of bone removal, this procedure requires more time.

As pinpoint decompression of the responsible lesion is enabled, this method might have advantages with respect to postoperative instability. ${ }^{[19]}$ Eun et al. ${ }^{[21]}$ showed that there is less chance of instability in patients with PED compared with open lumbar microdiscectomy. The advantage of PELL is that the field of view on the opposite side is superior to that offered by microscopic surgery and MEL. First, the endoscope tip is close to the objective, the endoscopic view is enlarged, and the fact that the operation is performed under irrigation using physiological saline ensures that the field of view is clear. Second, the viewpoint is located beyond the midline structures that disturb the field of view during other procedures. Therefore, the operator can clearly view the area, and even that of the opposite nerve root 
[Figure 3]. However, the fact that only limited kinds of instrumentation can be used during PELL tends to make operations more difficult. Drilling of the lateral recess can be carried out relatively easily. Although the corresponding top part of the lamina should be drilled before insertion of the endoscope, the ligament must be dissected from the surrounding bone. Complete detachment of the ligament on the opposite side is particularly difficult. If the detachment is difficult, the bone area attaching to the ligament should be drilled to remove the ligament together with the surrounding bone.

The technical difficulty of PELL using a single-port endoscope has already been described, as have the facts that additional time is required for the procedure and the end result might be incomplete decompression. ${ }^{[19]}$ Other studies have also emphasized that the learning curve for PELL is more gradual than those for MEL or PED. This technique should not be started without first achieving a certain level of proficiency in PED IL. Unless adequate technical skills have been acquired, the procedure can hardly be described as minimally invasive.

Some studies have described a method involving use of a second port to increase the number of instruments that can be used and remove the restrictions on field of view and usable instrumentation. ${ }^{[19,20,22]}$ In arthroscopy, surgery is performed using the triangle technique. Different reports have detailed the application of this technique to the spinal field and use different terms; irrigation endoscopic decompressive laminotomy (IEDL), ${ }^{[19]}$ percutaneous biportal endoscopic decompression (PBED), ${ }^{[20]}$ and two-portal percutaneous endoscopic decompression. ${ }^{[22]}$ In all these techniques, the arthroscope is inserted via the first port and the instrumentation is inserted via the second port, with the operation being performed under irrigation. All authors concluded that these methods resolve the restrictions on the size of instrument that can be inserted via the second port, enabling the use of drills, Kerison punches, and curettes employed in conventional laminectomy. In addition, no special equipment is required, as the surgery can be performed using an arthroscope and normal spinal surgery instrumentation. However, these secondportal technique may also need special training. ${ }^{[22]}$ Eum et al. ${ }^{[20]}$ reported that this technique is similar to a knee arthroscopic surgery or thoracoscopic surgery. All of these techniques used arthroscope but now we can use the single port endoscope that can allow using the instrumentation through the endoscope. Then the modification might be able to apply the second port method. Basically single port method is applied. And second port might be able to use for only the large instrumentation like Kerison punch that is used for the conventional surgery. Despite the requirement for technical proficiency described above, adaptations like this may offer one possible direction for the development of this method.

In conclusion, PELL is a method of treatment that minimizes soft-tissue damage caused by invasion associated with the surgical approach. However, because of its technical difficulty, PELL has not yet been become a popular procedure. Nevertheless, this technique offers a range of advantages if the operator acquires a sufficient level of technical proficiency to complete the procedure in approximately the same time required for microscopic lumbar laminotomy. Further developments in the procedure will be required to encourage more widespread use, such as using a larger endoscope to enable the use of a wider range of instrumentation, or use of a second port.

\section{Authors' contributions}

Study conception and design, performing the surgical technique: Y. Ohara

Critical revision: S. Shimizu, J. Mizuno

\section{Financial support and sponsorship}

None.

\section{Conflicts of interest}

There are no conflicts of interest.

\section{Patient consent}

Obtained.

\section{Ethics approval}

The ethics committee of ShinYurigaoka General Hospital approved this retrospective study.

\section{REFERENCES}

1. Ruan W, Feng F, Liu Z, Xie J, Cai L, Ping A. Comparison of percutaneous endoscopic lumber discectomy versus open lumbar microdiscectomy for lumbar disc herniation: a metaanalysis. Int $J$ Surg 2016;31:86-92.

2. Kleinpeter G, Markowitsch MM, Bock F. Percutaneous endoscopic lumbar discectomy: minimally invasive but perhaps only minimally usefil? Surg Neurol 1995;43:534-9.

3. Sairyo K, Egawa H, Matsuura T, Takahashi M, Higashino K, Sakai T, Suzue N, Hamada D, Goto T, Takata Y, Nishisho T, Goda Y, Sato R, Tsutsui T, Tonogai I, Kondo K, Tezuka F, Mineta K, Sugiura K, Takeuchi M, Dezawa A. State of the art: transforaminal approach for percutaneous endoscopic lumbar discectomy under local anesthesia. $J$ Med Invest 2014;61:217-25.

4. Sairyo K, Matsuura T, Higashino K, Sakai T, Takata Y, Goda Y, Suzue N, Hamada D, Goto T, Nishisho T, Sato R, Tsutsui T, Tonogai I, Mineta $\mathrm{K}$. Surgery related complications in percutaneous endoscopic lumbar 
discectomy under local anesthesia. J Med Invest 2014;61:264-9.

5. Hirano $\mathrm{Y}$, Mizuno J, Takeda M, Itoh Y, Matsuoka H, Watanabe K. Percutaneous endoscopic lumbar discectomy - early clinical experience. Neurol Med Chir 2012;52:625-30.

6. Yeung AT, Tsou PM. Posterolateral endoscopic exciseon for lumbar disc herniateon: surgical technique, outcome, and complications in 307 consecutive cases. Spine 2002;27:722-31.

7. Yeung AT, Yeung CA. Minimally invasive techniques for the management of lumbar disc herniation. Orthop Clin North Am 2007;38:363-72.

8. Sencer A, Yorukoglu AG, Akcakaya MO, Aras Y, Aydoseli A, Boyali O, Sencan F, Sabanci PA, Gomleksiz C, Imer M, Kiris T, Hepgul $\mathrm{K}$, Unal OF, Izgi N, Canbolat AT. Fully endoscopic interlaminar and transforaminal lumbar discectomy: short-term clinical results of 163 surgically treated patients. World Neurosurg 2014;82:884-90.

9. Reutten S, Komp M, Godolias G. An extreme lateral acess for the surgery of lumbar disc herniations inside the spinal canal using full-endoscopic uniportal transforaminal approach-technique and prospective results of 463 patients. Spine 2005:30:2570-8.

10. Jang JS, An SH, Lee SH. Transforaminal percutaneous endoscopic discectomy in the treatment of foraminal and extraforaminal lumbar disc herniations. J Spinal Disord Tech 2006;19:338-43.

11. Choi G, Kang HY, Modi HN, Prada N, Nicolau RJ, Joh JY, Pan WJ, Lee SH. Risk of developing seizure after percutaneous endoscopic lumbar discectomy. J Spinal Disord Tech 2011;24:83-92.

12. Dezawa A, Sairyo K. New minimally invasive endoscopic discectomy technique through the interlaminar space using a percutaneous endoscope. Asian J Endosc Surg 2011;4:94-8.

13. Wiltse LL, Kirkaldy-Willis WH, McIvor GW. The treatment of spinal stenosis. Clin Orthop Relat Res 1976;83-91.

14. Costa F, Sassi M, Cardia A, Ortolina A, De Santis A, Luccarell G, Fornari M. Degenerative lumbar spinal stenosis: analysis of the results in a series of 374 patients treated with unilaterall laminotomy for bilaterall microdecompression. J Neurosurg Spine 2007;7:579-86.

15. Yagi M, Okada E, Ninomiya K, Kihara M. Postoperative outcome after modified unilateral-approach microendoscopic midline decompression for degenerative spinal stenosis. $J$ Neurosurg Spine 2009;10:293-9.

16. Sairyo K, Sakai T, Higashino K, Inoue M, Yasui N, Dezawa A. Complications of endoscopic lumbar decompression surgery. Minim Invasive Neurosurg 2010;53:175-8.

17. Ikuta K, Tono O, Tanaka T, Arima J, Nakano S, Sasaki K, Oga K. Surgical complications of microendoscopic prodedures for lumbar spinal stenosis. Minim Invasive Neurosurg 2007;50:145-9.

18. Podichetty VK, Spears J, Isaacs RE, Booher J, Biscup RS. Complications associated with minimally invasive decompression for lumbar spinal stenosis. J Spinal Disord Tech 2006;19:161-6.

19. Soliman HM. Irrigation endoscopic decompression laminotomy. A new endoscopic approach for spinal stenosis decompression. Spine $J$ 2015; $15: 2282-9$

20. Eum JH, Hao DH, Son SK, Park CK. Percuaneous biportal endoscopic decompression for lumbar spinal stenosis: a technical note and prelim clinical results. J Neurosurg Spine 2016;24:602-7.

21. Eun SS, Lee SH, Sabai LA. Long-term follow-up results of percutaneous endoscopic lumbar discectomy. Pain Physician 2016;19:E1161-6.

22. Torudom Y, Dilokhuttakarn T. Two portal endoscopic decompression for lumbar spinal stenosis: prelim study. Asian Spine J 2016;10:335-42. 